ARTICLE

\title{
Spectromicroscopic insights for rational design of redox-based memristive devices
}

\author{
Christoph Baeumer', Christoph Schmitz', Amr H. H. Ramadan², Hongchu Du1,3, Katharina Skaja1, \\ Vitaliy Feyer ${ }^{1}$, Philipp Müller ${ }^{1}$, Benedikt Arndt ${ }^{1}$, Chun-Lin Jia ${ }^{1,3}$, Joachim Mayer ${ }^{1,3}$, Roger A. De Souza ${ }^{2}$, \\ Claus Michael Schneider ${ }^{1}$, Rainer Waser ${ }^{1,4} \&$ Regina Dittmann ${ }^{1}$
}

The demand for highly scalable, low-power devices for data storage and logic operations is strongly stimulating research into resistive switching as a novel concept for future non-volatile memory devices. To meet technological requirements, it is imperative to have a set of material design rules based on fundamental material physics, but deriving such rules is proving challenging. Here, we elucidate both switching mechanism and failure mechanism in the valence-change model material $\mathrm{SrTiO}_{3}$, and on this basis we derive a design rule for failure-resistant devices. Spectromicroscopy reveals that the resistance change during device operation and failure is indeed caused by nanoscale oxygen migration resulting in localized valence changes between $\mathrm{Ti}^{4+}$ and $\mathrm{Ti}^{3+}$. While fast reoxidation typically results in retention failure in $\mathrm{SrTiO}_{3}$, local phase separation within the switching filament stabilizes the retention. Mimicking this phase separation by intentionally introducing retention-stabilization layers with slow oxygen transport improves retention times considerably.

\footnotetext{
${ }^{1}$ Peter Gruenberg Institute, Forschungszentrum Juelich GmbH and JARA-FIT, 52425 Juelich, Germany. ${ }^{2}$ Institute of Physical Chemistry, RWTH Aachen University and JARA-FIT, 52056 Aachen, Germany. ${ }^{3}$ Ernst Ruska-Centre, Forschungszentrum Juelich GmbH and RWTH Aachen University, 52425 Juelich, Germany. ${ }^{4}$ Institute of Materials in Electrical Engineering and Information Technology II, RWTH Aachen University, 52056 Aachen, Germany.

Correspondence and requests for materials should be addressed to C.B. (email: c.baeumer@fz-juelich.de).
} 
$\mathrm{D}$ riven by the technological need for novel materials and innovative device concepts for future data storage and logic circuits, researchers in industry and academia are investigating intensively memristive transition metal oxides. This interest is due to the materials' potential use in future non-volatile memory or as novel neuromorphic circuits, even allowing for a local synergy of logic and memory ${ }^{1-6}$. The mechanism of resistive switching in transition metal oxides is widely accepted to be a nanoscale redox reaction, induced by oxygen-ion migration, the so-called valence-change mechanism ${ }^{7-12}$. During the SET processes, oxygen vacancies, which act as donor dopants and contribute electrons into the conduction band, form a so-called switching filament. The resulting local dopant distribution modifies the Schottky barrier at the interface with the top electrode and leads to a lower device resistance, the low-resistance state (LRS). RESET operations with opposite bias recover the high-resistance state (HRS) by disrupting or reoxidizing the switching filament ${ }^{13}$. Many research groups in academia and industry have already presented devices with a comparably simple architecture and excellent device performance. The ultimate aim regarding device performance is high uniformity for all devices on a single chip, an endurance (write cyclability) of at least $10^{7}$ cycles, scalability down to the nanometre regime and the potential for multibit operation ${ }^{3,5}$. Most of all, the ultra-nonlinear switching kinetics (also called voltage-time dilemma) between extremely fast switching times ( $\leq 10-100 \mathrm{~ns})$ and long retention times (exceeding 10 years) has to be met for non-volatile memory applications ${ }^{3}$.

While excellent retention has been demonstrated for many binary and ternary oxides in conjunction with fast switching, Noman et al. ${ }^{14}$ showed that in the absence of internal electric fields, the model valence-change material $\mathrm{SrTiO}_{3}$ cannot exhibit both properties simultaneously. In fact, retention failure after short times was reported for the LRS in single-crystalline $\mathrm{SrTiO}_{3}$ (refs 15-17). Polycrystalline and single-crystalline $\mathrm{SrTiO}_{3}$ films with considerable amounts of extended defects, on the other hand, exhibit much better retention behaviour ${ }^{16,18}$. On the basis of simulations of the $I-V$ characteristics and retention times, one finds that the retention failure mechanism for the LRS is based on the rupture of conducting filaments caused by reoxidation due to oxygen diffusion from the side ${ }^{19,20}$ or along the vertical direction ${ }^{21}$. Recent studies on the technologically most relevant systems of $\mathrm{HfO}_{2-x}$ (refs 22-26) and $\mathrm{Ta}_{2} \mathrm{O}_{5-x}$ (refs 19,20,27,28), however, reveal that extremely high retention times can be achieved with oxidizable electrodes or interlayers (the so-called oxygen-scavenging layer). Phenomenologically, this finding was attributed to the stability of certain oxygen distributions in the layer stack and filaments with sufficient oxygen-vacancy concentrations to be stable against reoxidation due to oxygen diffusion in the lateral direction. Experimental evidence for the role of local redox reactions for both the device function (resistance change between LRS and HRS) and for the retention failure, however, remains elusive so far, while some qualitative conclusions from these simulation-based studies ${ }^{20,21,29}$ such as the importance of the filament size and the oxygen-vacancy concentration within the filament and the adjacent layers certainly hold true.

Here we will experimentally demonstrate that one decisive (but previously overlooked) factor for the retention of memristive devices is the presence or formation of an oxygen-migrationblocking layer. We will show that for a retention-failure-resistant valence change in the model material $\mathrm{SrTiO}_{3}$, a local phase separation into a switching element and an artificial retentionstabilization layer is necessary to obtain the required nonlinearity of the kinetics. Single-crystalline $\mathrm{SrTiO}_{3}$ was chosen as the model material due to its homogeneity, the absence of grain boundaries as well as the extensive existing knowledge about defect chemistry, oxygen diffusion and cation diffusion available in literature ${ }^{30-36}$. Using spectromicroscopy, we explicitly confirm that the resistance changes that occur during device operation and retention failure are caused by oxygen migration and corresponding localized redox reactions between $\mathrm{Ti}^{3+}$ and $\mathrm{Ti}^{4+}$ configurations. We use these insights to demonstrate a new design rule for improved retention times in $\mathrm{SrTiO}_{3}$ devices: the intentional insertion of a retention-stabilization layer. The crucial parameter of the retention-stabilization layer is its capability to impede oxygen migration, thus protecting the switching filament from reoxidation. This finding paves the way for a rational design of reliable memristive cells for future memory or logic applications through the judicious choice of the oxygen-scavenging layer or intentional retention-stabilization layer.

\section{Results}

Spectromicroscopic analysis of resistance changes in $\mathrm{SrTiO}_{3}$. To visualize switching-induced local changes of the electronic and chemical structures of $\mathrm{SrTiO}_{3}$, we investigated memristive $\mathrm{Au} / \mathrm{SrTiO}_{3}(2 \mathrm{~nm}) / \mathrm{Nb}: \mathrm{SrTiO}_{3}$ devices (Fig. 1a) using X-ray photoemission electron microscopy (XPEEM, see the Methods section for fabrication details). For this purpose, multiple devices on the same chip were switched between the HRS $\left(\sim 10^{7}-10^{8} \Omega\right)$ and the LRS $\left(\sim 10^{3}-10^{4} \Omega\right)$ several times using a current compliance of $50 \mathrm{~mA}$ as shown in Fig. 1b. No separate forming step was necessary: the first application of positive voltages (SET process) led to a so-called soft-forming, which was very similar to subsequent SET processes. The resistance of each device was monitored over a period of several days (Fig. 1c). While the HRS was stable for each device investigated here, two classes of retention characteristics of the LRS were observed: for most devices in the LRS, the resistance increased by several orders of magnitude, indicative of a retention failure, while some devices $(\sim 20 \%)$ retained a resistance below $10^{5} \Omega$. In the following, these devices will be referred to as devices with a retention failure and devices with stable retention, respectively.

In a first step, the devices with stable retention were used to examine the valence-change mechanism, which is expected to be responsible for the resistive switching in $\mathrm{SrTiO}_{3}$. After delamination of the top electrode (see Methods), XPEEM imaging of a representative device with stable retention revealed a switching filament with enhanced contrast (Fig. 1d). As the photon energy $h v=458.5 \mathrm{eV}$ used in this case corresponds to the $\mathrm{Ti} \mathrm{L}_{3}$ absorption edge, one may surmise that the contrast arises because of a reduced Ti valence state inside this filament, which has been expected to be the origin of the low resistance $3,9,11,37,38$. In fact, extracting Ti L-edge absorption spectra for a region of interest within this filament and for a reference area from the surrounding reveals a strong contribution of $\mathrm{Ti}^{3+}$ within the filament, indicated by the dashed line, while the surrounding device area only shows $\mathrm{Ti}^{4+}$ contributions, as is expected for stoichiometric $\mathrm{SrTiO}_{3}$ (Fig. 1e) ${ }^{11,39}$. The local confinement of this valence change is evident from the false colour map of the $\mathrm{Ti}^{3+}$ contribution for the entire device area as shown in Fig. 1f: only for the filament area does one find a significant contribution. Since $\mathrm{Ti}^{3+}$ states in $\mathrm{SrTiO}_{3}$ correspond to electrons in the conduction band ${ }^{3}$, this region can in fact be regarded as the switching filament. We thus conclude that the resistive switching occurs within this filament due to a local reduction of the Ti.

While a locally confined, chemically reduced component has been previously observed after electroforming $7,10,11,40,41$ for different oxide materials, the different valence states between LRS and HRS have not been demonstrated until now. 
We therefore show that the observed $\mathrm{Ti}^{3+}$ valence change in our samples is reversible: for a device that was switched back to the HRS, no $\mathrm{Ti}^{3+}$ spectral contributions could be detected (Supplementary Fig. 1) although XPEEM images indicate the presence of a locally modified area, which we ascribe to the reoxidized switching filament. In other words, we have demonstrated explicitly that field-driven local reduction and reoxidation indeed constitute the fundamental mechanism behind resistive switching in this model system.

As discussed in the introduction, reoxidation of the switching filament, albeit on a different (longer) timescale, is also believed to be responsible for the retention failure of the LRS. We analysed, therefore, the local electronic structure of devices with retention failure, as shown for an exemplary device in Fig. 1g-i. While the XPEEM images show a small area with a darker contrast, which might be the remainder of a switching filament, the Ti L-edge absorption spectra for this region and the surroundings, and in particular the false colour map of $\mathrm{Ti}^{3+}$, reveal that no significant $\mathrm{Ti}^{3+}$ contribution is present in the entire device area. As the $\mathrm{SrTiO}_{3}$ film is only $2 \mathrm{~nm}$ thin, which is significantly smaller than the probing depth of our XPEEM measurements $(\sim 5 \mathrm{~nm})$, this means that the initially reduced switching filament is completely reoxidized during retention failure.
The role of oxygen migration for the retention of $\mathrm{SrTiO}_{3}$-based devices is also directly apparent from the comparison of the retention characteristics in vacuum with the behaviour in ambient atmosphere (Supplementary Fig. 2). The memory window (ratio between HRS and LRS) after $5 \times 10^{5} \mathrm{~s}$ in vacuum is one order of magnitude higher than for samples kept in air. The retention times are improved because no oxygen is available for reoxidation of the switching filament from the environment. Consequently, these results experimentally verify the hypotheses of previous simulation-based models ${ }^{21}$ that propose the retention failure of the LRS to be due to oxygen diffusion along the vertical direction. Given that the reoxidation is inhibited in vacuum, oxygen species necessary for retention failure are evidently not supplied from the lateral direction. Instead, the oxygen species are apparently stored at the interface between the top electrode and the $\mathrm{SrTiO}_{3}$ film, inside the top electrode (for example, within grain boundaries), or even supplied from the surrounding atmosphere. We can thus conclude that incorporation and vertical diffusion of oxygen from the top electrode or surrounding into $\mathrm{SrTiO}_{3}$ plays a dominant role in the retention-failure mechanism.

As the reoxidation of switching filaments evidently needs to be inhibited to protect a device from retention failure, the question
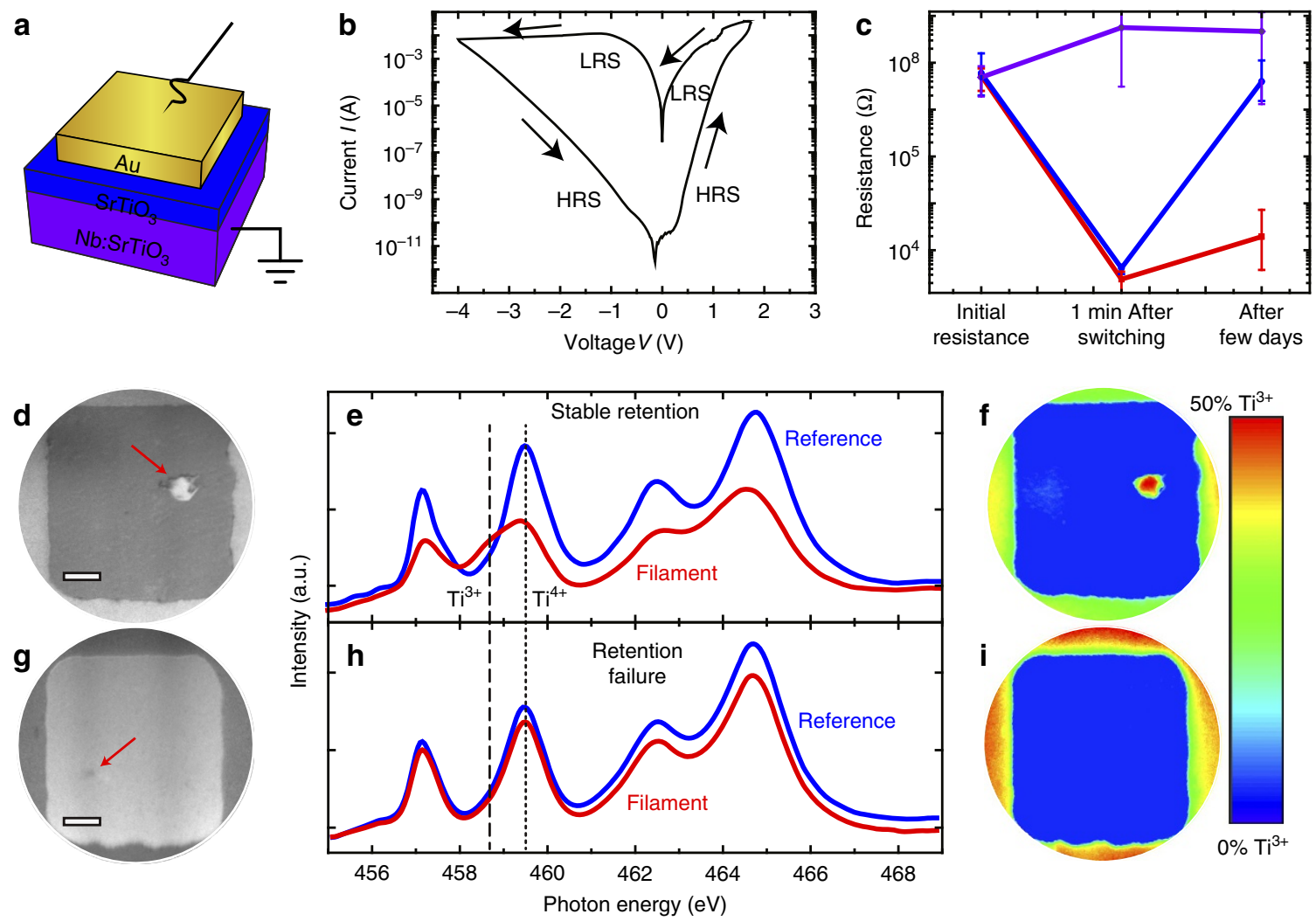

Figure 1 | Ti L-edge XPEEM analysis of $\mathrm{SrTiO}_{\mathbf{3}}$ memristive devices. (a) Device schematic. (b) Representative $I-V$ curve. (c) Retention behaviour of different devices on the same chip. Devices RESET into the HRS remain at a constant resistance over several days (violet curve). Devices that were SET into the LRS show two classes of retention behaviour: stable retention and retention failure (red and blue curves, respectively). For each class of retention behaviour, the resistance was averaged for five representative devices. Error bars indicate the minimum and maximum values obtained for each resistance state. (d) XPEEM image of a device with a stable retention recorded with a photon energy of $458.5 \mathrm{eV}$. Filament indicated by a red arrow. Scale bar, $2 \mu \mathrm{m}$. (e) Ti L-edge spectra extracted from the XPEEM image stack for a region inside the bright filament discernable in $\mathbf{d}$ (red line) and for the surrounding device area (blue line). The dotted line and the dashed line denote the $e_{g}$ level of the $\mathrm{Ti}_{3}$ edge in $\mathrm{Ti}^{4+}$ and $\mathrm{Ti}^{3+}$ configuration, respectively. (f) False colour map of the $\mathrm{Ti}^{3+}$ contribution for the device in $\mathbf{d}$. Only within the suspected switching filament, there is a significant spectral contribution of $\mathrm{Ti}^{3+}$. The enhanced contrast surrounding the active device is caused by the reactive ion beam etching involved in the electrode structuring and is not associated with the device conductance. ( $\mathbf{g}$ ) XPEEM image of a device with retention failure recorded with a photon energy of $459.5 \mathrm{eV}$. (h) Ti L-edge spectra extracted from the XPEEM image stack for a region inside the suspected switching filament discernable in $\mathbf{g}$ (red line) and for the surrounding device area (blue line). (i) False colour map of the $\mathrm{Ti}^{3+}$ spectral contribution for the device in $\mathbf{g}$. No $\mathrm{Ti}^{3+}$ contribution is discernible for the entire device area. 
arises why some devices on the same chip show stable retention, while most devices exhibit retention failure. Given the comparably fast ambipolar diffusion of oxygen vacancies in $\mathrm{SrTiO}_{3}$ (refs 30-32), we would predict retention failure for every device $^{14}$. To elucidate the microscopic mechanism for retention stabilization, we carefully analysed the local chemical configuration of the switching filaments of the devices shown in Fig. 1. O -K-edge absorption spectra for the filament and the surroundings of the device with stable retention reveal a strong modification of the local environment of the $\mathrm{O}$-ions within the filament (Fig. 2a). From comparison with literature, we assume that this modification stems from a significant $\mathrm{SrO}$ contribution $^{42}$, which is plausible as the formation of $\mathrm{SrO}$ islands on the surface of $\mathrm{SrTiO}_{3}$ has been previously observed after high-temperature treatments exceeding $1,000^{\circ} \mathrm{C}$ (refs $43-45$ ). It has already been shown that Joule heating during electrical treatment can provide sufficiently high temperatures to promote such a phase separation ${ }^{9,11,46}$. The false colour map of the SrO contribution to the $\mathrm{O}-\mathrm{K}$-edge shown in Fig. $2 \mathrm{~b}$ reveals that this modified O-environment is only present within the switching filament for the device with stable retention and the device in the HRS (Supplementary Fig. 1), while it is absent for the device with retention failure (Fig. $2 \mathrm{c}, \mathrm{d}$ ). We thus propose that in the first case, significant Joule heating within the filament leads to a localized phase separation into a Sr-deficient $\mathrm{SrTiO}_{3}$ and a $\mathrm{SrO}$ interface component, which becomes a surface component after delamination. As SrO surface components have been detected using X-ray photoelectron spectroscopy (XPS) ${ }^{46,47}$ before, we performed energy-resolved XPEEM for both devices for the Sr 3d level to verify the anticipated phase separation. As typically observed for $\mathrm{SrTiO}_{3}$ thin films, the $\mathrm{Sr}$ 3d spectra extracted from these scans can be fitted well with a doublet for the bulk contribution and an additional doublet found at $0.8 \mathrm{eV}$ higher binding energy, which corresponds to SrO-type surface components (Fig. 2e,f) ${ }^{46,47}$. As expected, the amount of this surface component is significantly higher within the filament of the device with stable retention, as is evident from the representative spectra for the filament and the surrounding and the false colour map of the SrO contribution shown in Fig. $2 \mathrm{e}-\mathrm{g}$. The electron kinetic energy used here was around $60-70 \mathrm{eV}$, corresponding to the minimum of the kinetic-energy-dependent inelastic mean free path of just $0.5 \mathrm{~nm}$ (ref. 48). The resulting surface sensitivity of the XPS analysis thus supports the hypothesis that the observed phase separation for devices with stable retention yields a Sr-deficient filament with a SrO layer in the surface region.

As for the $\mathrm{O}$-K-edge, there is no indication of this local phase separation in the XPS analysis for the device with retention failure (Fig. $2 \mathrm{~h}-\mathrm{j}$ ). Consequently, it appears that the phase separation into a Sr-deficient $\mathrm{SrTiO}_{3}$ and a $\mathrm{SrO}$ surface layer is mandatory for stable retention. This is further corroborated by a comparison of all measured devices: four devices with retention failure and four devices with stable retention were investigated with XPEEM. For all of these devices, we observed no $\mathrm{Ti}^{3+}$ or $\mathrm{SrO}$ spectral contribution for the devices with retention failure, while all devices with stable retention exhibited a filament with a $\mathrm{Ti}^{3+}$ contribution and an $\mathrm{SrO}$ interface component. Intuitively, this phase separation could be presumed to be responsible for strongly suppressed reoxidation of the switching filament from the vertical direction due to impeded oxygen diffusion within and through the SrO layer. Reoxidation from the side is unlikely to contribute significantly due to the filament dimensions, which would require $\mu \mathrm{m}$ diffusion lengths from the side in contrast to only $2 \mathrm{~nm}$ in the vertical direction. The diffusion of oxygen in SrO, however, is not well understood. A theoretical description of oxygen migration in this system will therefore be provided below. Beforehand, we will
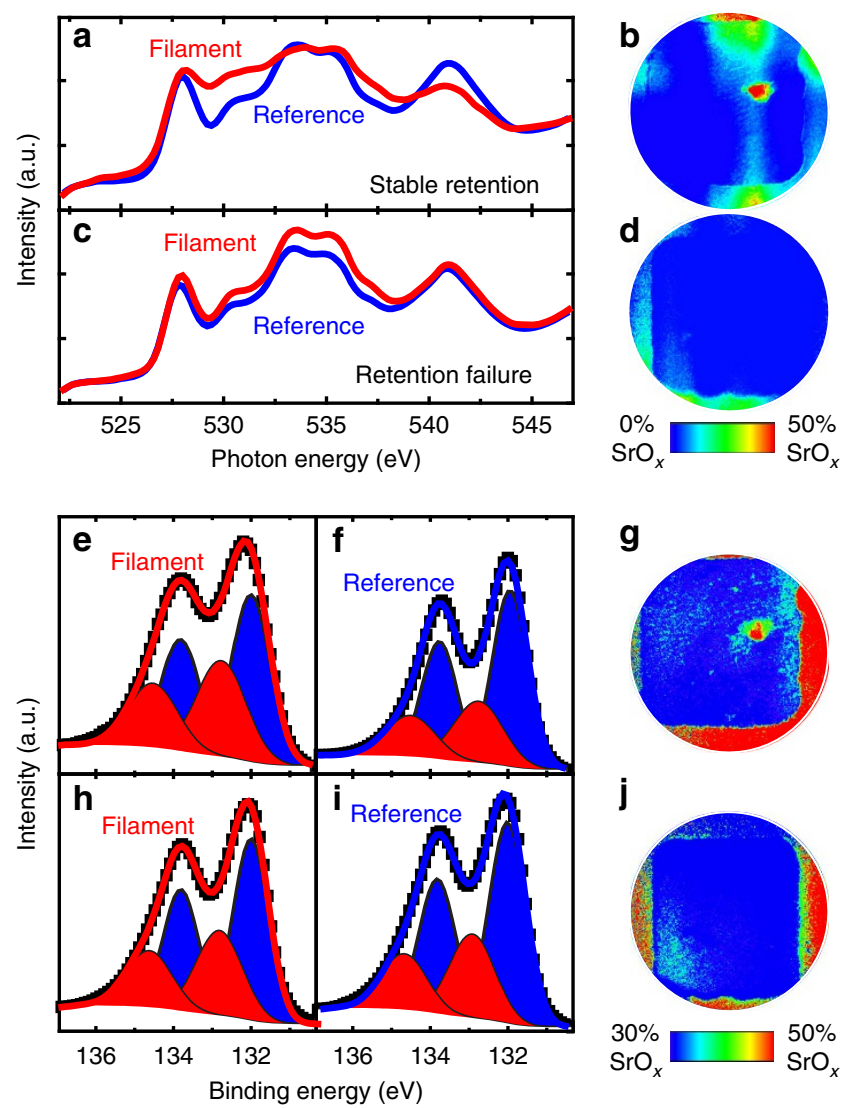

g

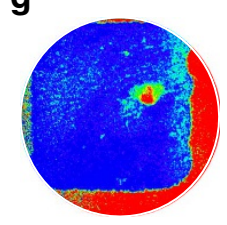

j

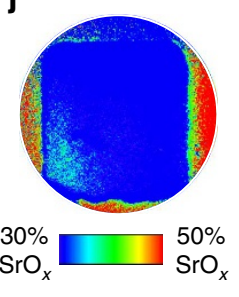

Figure 2 | 0 -K-edge and Sr 3d XPEEM analysis of $\mathrm{SrTiO}_{3}$ memristive devices. (a) O-K-edge spectra extracted from the XPEEM image stack for the switching filament (red line) and for the surrounding device area (blue line) for the device with stable retention (the same device as in Fig. 1d-f). (b) False colour map of the $\mathrm{SrO}$ contribution for the same device. Only within the suspected switching filament, there is a significant spectral contribution of $\mathrm{SrO}$. The apparent vertical stripe in the centre of the device is an artefact from the uncorrected but small horizontal energy dispersion of the NanoESCA energy filter. The resulting contrast is much lower, however, than the real contrast detected within the filament. (c) O-K-edge spectra extracted from the XPEEM image stack for a region within the switching filament (red line) and for the surrounding device area (blue line) for the device with retention failure (the same device as in Fig. 1g-i). (d) False colour map of the $\mathrm{SrO}$ contribution for the same device. No significant contribution of $\mathrm{SrO}$ can be detected. (e) Sr 3d spectrum for the switching filament (black data points) for the device with stable retention with a fit (red line) consisting of a bulk component (blue doublet) and a SrO-like surface component (red doublet). (f) $\mathrm{Sr} 3 \mathrm{~d}$ spectrum extracted from a region surrounding the switching filament (black data points) for the same device with a similar fit (blue line). The SrO-like surface component is suppressed in comparison with e. (g) False colour map of the $\mathrm{SrO}$ contribution for the same device. Only within the suspected switching filament, there is a significant contribution of $\mathrm{SrO}$. (h) $\mathrm{Sr} 3 \mathrm{~d}$ spectrum for the suspected switching filament (black data points) for the device with retention failure with a similar fit (red line). (i) Sr 3d spectrum extracted from a region surrounding the switching filament (black data points) for the same device and a similar fit (blue line). The SrO-like surface component is equally small in both spectra. (j) False colour map of the $\mathrm{SrO}$ contribution for the same device. No significant contribution of $\mathrm{SrO}$ can be detected.

have to consider the question of whether the observed phase separation is a side effect of the process leading to stable retention, or indeed its origin. To demonstrate and make use of the influence of the $\mathrm{SrTiO}_{3} / \mathrm{SrO}$ interface for the device 
performance, we investigated the retention characteristic of an intentionally created interface between $\mathrm{Sr}$-deficient $\mathrm{SrTiO}_{3}$ and SrO.

Stable retention behaviour in $\mathrm{SrTiO}_{3} / \mathrm{SrO}$ heterostructures. To mimic the phase separation described above, artificial $\mathrm{SrTiO}_{3} / \mathrm{SrO}$ heterostructures were created by successive pulsed laser deposition of $2 \mathrm{~nm} \sim 1$ at $\%$ Sr-deficient $\mathrm{SrTiO}_{3}$ and $1 \mathrm{~nm} \mathrm{SrO}$ (ref. 49). For the quantitative comparison of the retention behaviour of $\mathrm{SrTiO}_{3}$ thin-film devices as investigated in Fig. 1 with $\mathrm{SrTiO}_{3} / \mathrm{SrO}$ heterostructures, multiple devices were switched between the HRS and LRS configurations for both samples and the resistance values were monitored over several days. To reveal the retention behaviour of the as-prepared films rather than the characteristics of local phase separations as described above, the current compliances were chosen as low as possible such that the memory window was still larger than two orders of magnitude ${ }^{5,19}$. The $I-V$ hysteresis for both samples remained similar in shape, indicating that the same fundamental switching mechanism was responsible for the resistance change (Supplementary Fig. 3). The average resistance as a function of current compliance and time is shown in Fig. 3a,b. While the retention of the HRS was rather stable for each case, the LRS showed strong differences between both samples, depending on the current compliance. For $10 \mathrm{~mA}$, the average LRS increased to a value barely distinguishable from the HRS within a short
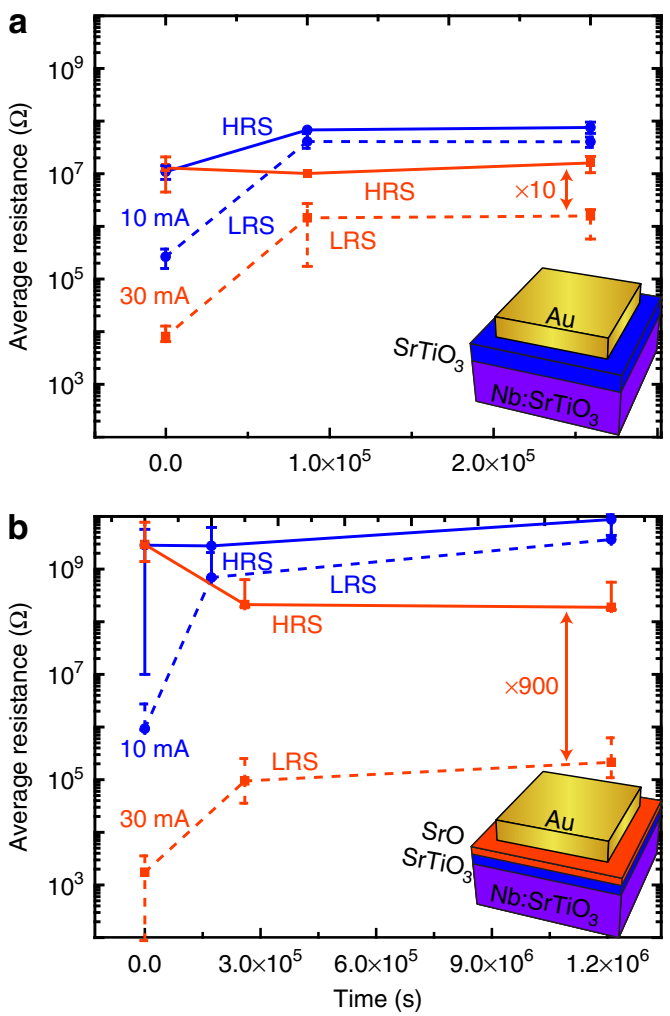

Figure 3 | Retention behaviour of $\mathrm{SrTiO}_{3}$ thin-film devices and $\mathrm{SrTiO}_{3} / \mathrm{SrO}$ heterostructures. (a) Average resistance of $\mathrm{SrTiO}_{3}$ thin-film devices in the LRS (dashed line) and the HRS (solid line) as a function of time after switching for a SET current compliance of $10 \mathrm{~mA}$ (blue lines) and $30 \mathrm{~mA}$ (red lines). The device schematic is shown in the inset. (b) Average resistance of $\mathrm{SrTiO}_{3} / \mathrm{SrO}$ heterostructure devices in the LRS (dashed line) and the HRS (solid line) as a function of time after switching for a SET current compliance of $10 \mathrm{~mA}$ (blue lines) and $30 \mathrm{~mA}$ (red lines). Error bars indicate the minimum and maximum values obtained for each resistance state. The device schematic is shown in the inset. time for both $\mathrm{SrTiO}_{3}$ thin-film devices and $\mathrm{SrTiO}_{3} / \mathrm{SrO}$ heterostructures. For $30 \mathrm{~mA}$, on the other hand, LRS and HRS are also very close to each other for $\mathrm{SrTiO}_{3}$ thin-film devices after $10^{5} \mathrm{~s}$ (or even earlier), but $\mathrm{SrTiO}_{3} / \mathrm{SrO}$ heterostructures exhibit a large memory window of roughly three orders of magnitude even after $10^{6} \mathrm{~s}$. While the data shown in Fig. 3 is averaged over multiple devices, we would like to note that in contrast to the $\mathrm{SrTiO}_{3}$ thin-film devices, we did not observe any device with a complete retention failure (HRS and LRS indistinguishable) for the $\mathrm{SrTiO}_{3} / \mathrm{SrO}$ heterostructures after switching with $30 \mathrm{~mA}$. This improved retention behaviour is caused by a rather stable LRS value after an initial relaxation combined with a stable HRS on the order of $10^{8} \Omega$. It was achieved with less power than was necessary for the observation of single-SrTiO $\mathrm{S}_{3}$ devices with stable retention (cf. Fig. 1), making it less likely that an additional phase separation is responsible for the stable retention. The dispensability of phase separations for good retention times also results in a more uniform behaviour of different devices on the same chip: while the devices shown in Figs 1 and 2 are vastly different in their retention behaviour, all devices containing the intentional $\mathrm{SrTiO}_{3} / \mathrm{SrO}$ interfaces exhibit a similar resistance for all times investigated here.

Oxygen migration in $\mathrm{SrTiO}_{3} / \mathrm{SrO}$ heterostructures. Given the significant retention improvement through the insertion of a $\mathrm{SrO}$ layer, we conclude that the Joule heating enabled phase separation into a switching element and an artificial retention-stabilization layer is not a side effect of high temperatures during forming. In fact, this phase separation is the origin of the observed stable retention for the device shown in Figs $1 d-f$ and 2a,b. We therefore now consider how this phase separation can assist in prolongating the retention times. As discussed above, a retention failure is caused by the reoxidation of the switching filament from the vertical direction. For the $\mathrm{SrTiO}_{3} / \mathrm{SrO}$ heterostructure, three different scenarios can prevent this reoxidation:

(i.) Oxygen storage capability. Similar to the oxygen exchange between different oxide layers in oxide dual-layer memory elements under applied electric fields ${ }^{50}$, oxygen from the $\mathrm{SrTiO}_{3}$ could be incorporated into $\mathrm{SrO}$ (as interstitials or into existing vacancies) during the SET operation. The SrO layer can be regarded as an oxygen reservoir. This may lead to a new (meta-)stable oxygen distribution between the two adjacent oxides eliminating the driving force for reoxidation of the $\mathrm{SrTiO}_{3}$ without applied electric fields and therefore lead to improved retention times.

(ii.) Surface reaction rate of oxygen incorporation. If the storage of additional oxygen in the retention-stabilization layer is impeded due to a lack of available lattice sites (no vacancies available and unfavourable energy of formation for oxygen interstitials), oxygen must be excorporated from the retention-stabilization layer during the SET operation, similar to the SET operation and reoxidation in $\mathrm{SrTiO}_{3}$ thin-film devices described above. In this case, reoxidation of the $\mathrm{SrTiO}_{3}$ during RESET or retention failure inevitably requires the incorporation and diffusion of additional oxygen into the heterostructure from the surroundings. Since conduction band electrons contribute to the ratedetermining step in the incorporation of oxygen into the oxide lattice ${ }^{51,52}$, this step might be impeded in $\mathrm{SrO}$ due to the comparably large bandgap (between 5 and $6.5 \mathrm{eV}$ ). The inhibited oxygen incorporation may therefore be responsible for the enhanced retention times of the heterostructures.

(iii.) Diffusion rate. At the same time, the oxygen needs to migrate from the top surface into the conductive filament within the $\mathrm{SrTiO}_{3}$ after the incorporation into the 
retention-stabilization layer. Given the fast diffusion rates in $\mathrm{SrTiO}_{3}$, the improved retention of the heterostructures may be caused by slower ambipolar diffusion rates within the $\mathrm{SrO}$. If the incorporation rate of the retention-stabilization layer was as fast as in $\mathrm{SrTiO}_{3}$, slow diffusion through the retention-stabilization layer may become the rate-determining step and thus retard the reoxidation of the switching filament.

To clarify which of the effects (i-iii) are responsible for the improved retention times, we first describe the oxygen migration in the heterostructure through static lattice simulations. Given that both pulsed laser deposition and phase separation during electrical treatments result in oxide layers with considerable amounts of point defects such as anion and cation vacancies, we analysed the migration of oxygen vacancies within $\mathrm{SrTiO}_{3} / \mathrm{SrO}$ heterostructures. In Fig. 4, we show the site and saddle-point energies corresponding to the migration of an oxygen vacancy through the heterostructure. One notices a considerable difference in the migration barriers in the two materials, with the barriers in $\mathrm{SrO}(\sim 1.2 \mathrm{eV})$ being twice as high as those in $\mathrm{SrTiO}_{3}$ $(\sim 0.6 \mathrm{eV})$. This suggests that, once oxygen ions are incorporated into $\mathrm{SrO}_{1-x}$ under an electric field (which lowers the barrier for migration), their return is hindered. There are also small differences in the site energies within $\mathrm{SrTiO}_{3}$ (this is due to the biaxial strain arising from lattice mismatch) and between $\mathrm{SrTiO}_{3}$ and $\mathrm{SrO}$ (suggesting a small amount of vacancy redistribution from $\mathrm{SrO}$ to $\left.\mathrm{SrTiO}_{3}\right)^{52}$.

On the basis of these calculations, we can conclude that impeded oxygen transport within the $\mathrm{SrTiO}_{3} / \mathrm{SrO}$ heterostructure (mechanism iii) could in fact lead to improved retention times. At the same time, we cannot exclude that mechanisms i and ii play a similar role in $\mathrm{SrO}$, as oxygen incorporation reactions and the defect structure in SrO are not well understood. We therefore now turn to model oxide materials to clarify whether the oxygen storage capability or impeded oxygen transport is a dominating

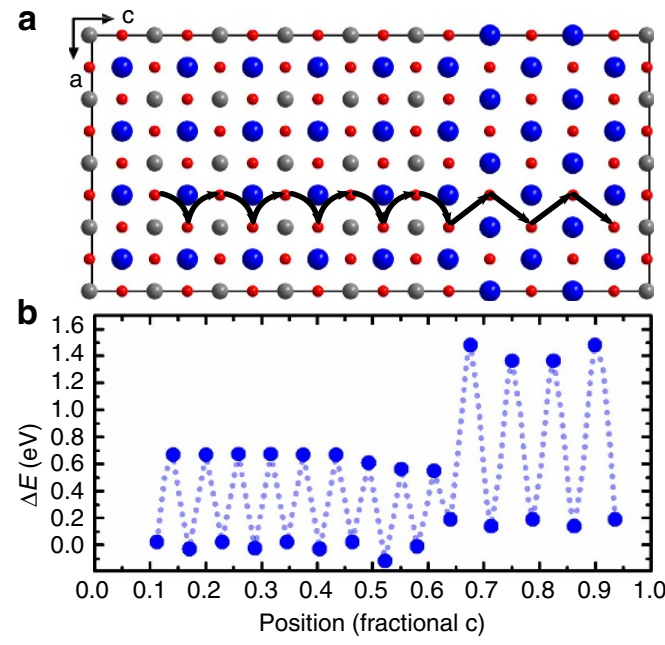

Figure 4 | Oxygen-vacancy migration in the $\mathrm{SrTiO}_{3} / \mathrm{SrO}$ heterostructure. (a) Illustration of the simulation cell of the heterostructure, with the simulated migration path indicated schematically by black arrows. Sr in blue, $\mathrm{Ti}$ in grey and $\mathrm{O}$ in red. (b) Energies of the sites and saddle points corresponding to an oxygen vacancy migrating through the heterostructure. The $x$ axis is scaled to the fractional coordinates of the $c$ axis of the simulation cell. All plotted energies are referenced to the average vacancy formation in the $\mathrm{SrTiO}_{3}$ regime of the simulation cell. The dotted line serves as a guide to the eye for the change in energy during the migration process. factor of retention improvement through the intentional insertion of retention-stabilization layers.

Rational design of retention-failure-resistant memristive devices. The broad variety of physical properties exhibited by oxides allows us to select prototypical materials for the retentionstabilization layer. For this purpose, we select yttria-stabilized $\mathrm{ZrO}_{2}$ (YSZ) and $\mathrm{Al}_{2} \mathrm{O}_{3}$. These oxides differ most severely in two specific properties: electronically, both $\mathrm{YSZ}$ and $\mathrm{Al}_{2} \mathrm{O}_{3}$ are rather inactive insulators with a large bandgap, but they are prototypical representatives for fast oxygen transport combined with high oxygen-vacancy concentration and slow oxygen transport combined with very low oxygen-vacancy concentration, respectively. While YSZ is the prototypical oxygen-ion-conducting electrolyte, with a measured oxygen diffusion coefficient of $D^{\mathrm{YSZ}}(500 \mathrm{~K}) \approx 10^{-13} \mathrm{~cm}^{2} \mathrm{~s}^{-1}$ and an activation enthalpy of just $1 \mathrm{eV}$ (ref. 53), $\mathrm{Al}_{2} \mathrm{O}_{3}$ exhibits low diffusion coefficients, even close to its melting point; the extrapolated value with an activation energy of $6.5 \pm 0.5 \mathrm{eV}$ (ref. 54) is $D^{\mathrm{Al}_{2} \mathrm{O}_{3}}(500 \mathrm{~K}) \approx 10^{-65} \mathrm{~cm}^{2} \mathrm{~s}^{-1}$. Due to the high concentration of oxygen vacancies, YSZ also exhibits a large amount of available lattice sites for oxygen storage, while $\mathrm{Al}_{2} \mathrm{O}_{3}$ exhibits very small deviations from nominal stoichiometry.

On the basis of these criteria, $\mathrm{SrTiO}_{3}(2 \mathrm{~nm}) / \mathrm{YSZ}(1 \mathrm{~nm})$ and $\mathrm{SrTiO}_{3}(2 \mathrm{~nm}) / \mathrm{Al}_{2} \mathrm{O}_{3}(1 \mathrm{~nm})$ heterostructures were fabricated by successive pulsed laser deposition. Multiple devices on each chip were switched between the HRS and the LRS using similar voltages as for the $\mathrm{SrTiO}_{3}$ thin-film devices. One may question whether the $\mathrm{YSZ}$ or $\mathrm{Al}_{2} \mathrm{O}_{3}$ layers might be actively involved in the resistive switching processes in these heterostructures. If the switching in these heterostructures was dominated by the inserted retention-stabilization layer, however, we would expect a different shape or even the opposite switching direction, as was demonstrated before for resistive switching in $\mathrm{Al}_{2} \mathrm{O}_{3}$ (ref. 55). Instead, we observed that the shape and polarity of the $I-V$ characteristics as well as the absolute voltages and currents, which are typically observed in these heterostructures, were still the same as for $\mathrm{SrTiO}_{3}$ thin-film devices (Supplementary Fig. 3). Given that the resulting LRS state of the heterostructures is orders of magnitude smaller than the initial resistance or the HRS from the $\mathrm{SrTiO}_{3}$ thin-film devices, we conclude that the resistance change in the heterostructures must occur in the $\mathrm{SrTiO}_{3}$ layer, although we cannot rule out that additional processes take place in the $\mathrm{YSZ}$ or $\mathrm{Al}_{2} \mathrm{O}_{3}$ layers. Impedance spectroscopy experiments performed on virgin cells and cells in the LRS and HRS confirmed that the resistive switching in the heterostructures also occurs within a confined filament (Supplementary Fig. 4). For $\mathrm{SrTiO}_{3} / \mathrm{YSZ}$ heterostructures, we observe switching with a large initial memory window with a current compliance of $1 \mathrm{~mA}$. For $10 \mathrm{~mA}$ current compliance, we observe a comparably low HRS of just $10^{7} \Omega$. Nevertheless, the initial memory window after switching with $10 \mathrm{~mA}$ current compliance is three orders of magnitude, which is similar to the memory windows obtained for the $\mathrm{SrTiO}_{3}$ thin-film devices. For even higher current compliances, we observe a device breakdown to a low resistance. $\mathrm{SrTiO}_{3} / \mathrm{Al}_{2} \mathrm{O}_{3}$ heterosturctures, on the other hand, can be switched reliably with 10 or $30 \mathrm{~mA}$ current compliance, resulting in similar initial memory windows of $10^{3}$ and $10^{4}$, respectively.

For closer inspection of the $\mathrm{SrTiO}_{3} / \mathrm{Al}_{2} \mathrm{O}_{3}$ heterostructure, we performed high-angle annular dark-field scanning transmission electron microscopy (HAADF-STEM) imaging of an electrically treated device, revealing epitaxial growth of the aluminium oxide on top of TiO-terminated $\mathrm{SrTiO}_{3}$. Figure $5 \mathrm{a}$ shows the crosssection HAADF-STEM images of the $\mathrm{SrTiO}_{3}(2 \mathrm{~nm}) / \mathrm{Al}_{2} \mathrm{O}_{3}$ 

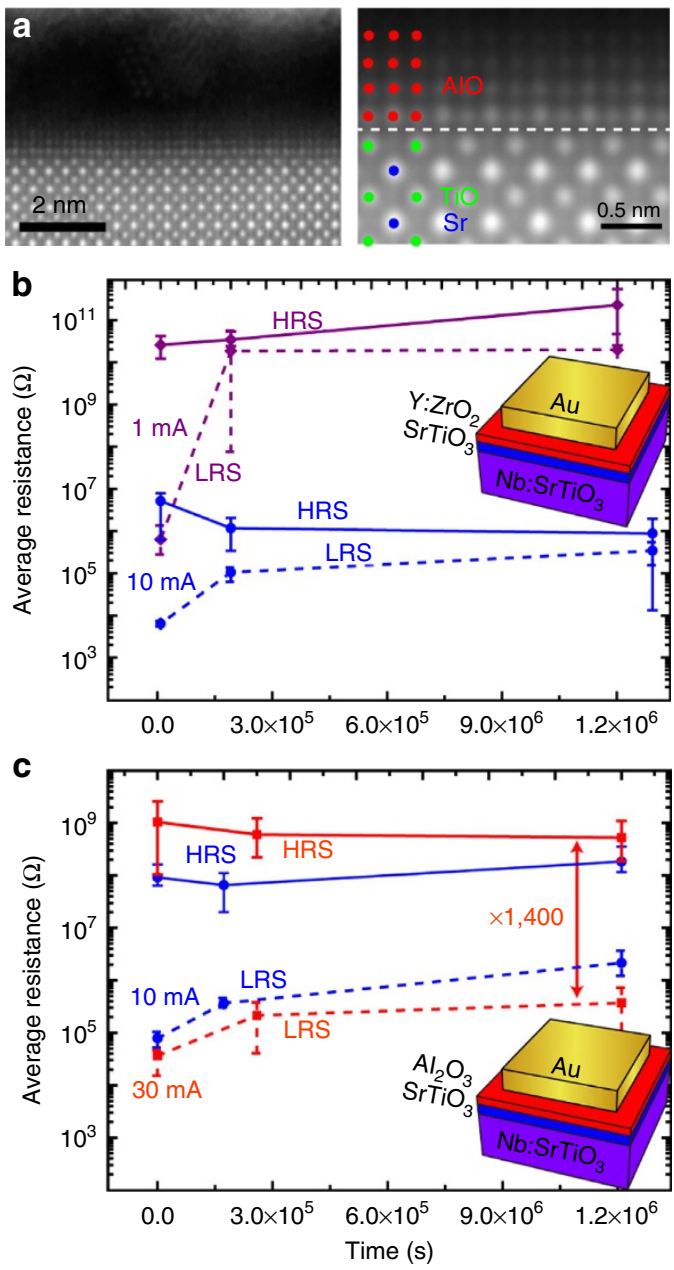

Figure 5 | Retention behaviour of $\mathrm{SrTiO}_{3} / \mathrm{YSZ}$ heterostructures and $\mathbf{S r T i O}_{\mathbf{3}} / \mathbf{A l}_{\mathbf{2}} \mathbf{O}_{\mathbf{3}}$ heterostructures. (a) Left: HAADF-STEM image of the epitaxial $\mathrm{SrTiO}_{3} / \mathrm{Al}_{2} \mathrm{O}_{3}$ interface (denoised by a nonlinear filter ${ }^{62}$ ); right: magnified one-dimensional averaged image better revealing the structure of the epitaxial interfaces. (b) Average resistance of $\mathrm{SrTiO}_{3} / \mathrm{YSZ}$ heterostructure devices in the LRS (dashed line) and the HRS (solid line) as a function of time after switching for a SET current compliance of $1 \mathrm{~mA}$ (violet lines) and $10 \mathrm{~mA}$ (blue lines). The device schematic is shown in the inset. (c) Average resistance of $\mathrm{SrTiO}_{3} / \mathrm{Al}_{2} \mathrm{O}_{3}$ heterostructure devices in the LRS (dashed line) and the HRS (solid line) as a function of time after switching for a SET current compliance of $10 \mathrm{~mA}$ (blue lines) and $30 \mathrm{~mA}$ (red lines). Error bars indicate the minimum and maximum values obtained for each resistance state. The device schematic is shown in the inset.

heterostructures from a switching filament, which was marked by conductive atomic force microscopy before the preparation of the TEM lamella (Supplementary Fig. 5). The observed atomic structure for the as-grown aluminium oxide is identical for the entire lamella (within the switching filament as well as few micrometres away from it) and appears to be similar to the reported $\mathrm{SrTiO}_{3} / \gamma-\mathrm{Al}_{2} \mathrm{O}_{3}$ in literature ${ }^{56,57}$. The $\mathrm{TiO} / \mathrm{AlO}$ interfaces are more evidently seen from the one-dimensional averaged HAADF-STEM image.

On the basis of this structural characterization, we assume that our films are indeed good representatives for fast and slow oxygen transport coupled with high and low oxygen storage capabilities, respectively. Comparing the retention characteristics of these heterostructures, therefore, allows the distinction between mechanisms $\mathrm{i}$ and ii-iii. In fact, we observe compelling differences: HRS and LRS values were similar to each other after short times $\left(<10^{5} \mathrm{~s}\right)$ for the case of the oxygen-conducting $\mathrm{SrTiO}_{3} / \mathrm{YSZ}$ heterostructure for all current compliances (Fig. 5b). For the oxygen-diffusion-suppressing $\mathrm{Al}_{2} \mathrm{O}_{3}$, on the other hand, stable retention of both HRS and LRS was obtained for both 10 and $30 \mathrm{~mA}$, with a large memory window of several orders of magnitude even after more than $10^{6} \mathrm{~s}$ (as shown for the average resistance in Fig. 5c). As was the case for the $\mathrm{SrTiO}_{3} / \mathrm{SrO}$ heterostructures, we did not observe retention failure in any $\mathrm{SrTiO}_{3} / \mathrm{Al}_{2} \mathrm{O}_{3}$ device. This is a marked improvement compared with devices fabricated without retention-stabilization layers or retention-stabilization layers with fast oxygen diffusion. Since these heterostructures exhibit an initial memory window of $10^{3}$ for switching with $10 \mathrm{~mA}$, which is nearly identical to the memory windows for $\mathrm{SrTiO}_{3} / \mathrm{YSZ}$ heterostructure or $\mathrm{SrTiO}_{3}$ thin-film devices, one can conclude that the observed stable retention cannot be explained by initially higher memory windows. We can now conclude, therefore, that inhibiting the back transport of oxygen (at room temperature and without applied electric fields) is the most effective method to stabilize retention in $\mathrm{SrTiO}_{3}$-based memristive devices. The oxygen storage capability (mechanism i) of the retention-stabilization layer does not appear to play an important role, as the introduction of YSZ layers does not improve retention. Instead, we must conclude from the observed effects that oxygen is excorporated from the oxide layers during the SET operation, and that the reoxidation of the switching filament can be prevented if no oxygen can enter the $\mathrm{SrTiO}_{3}$ (mechanisms ii and iii). As is evident from the improved retention with $\mathrm{SrO}$ and $\mathrm{Al}_{2} \mathrm{O}_{3}$ retention-stabilization layers, slow oxygen migration within the layer (mechanism iii) can protect the switching filament from fast reoxidation. Incorporation of such layers with slow oxygen diffusion for the protection of the switching filaments may therefore be regarded as a design rule for failure-resistant memristive devices. Since both $\mathrm{SrO}$ and $\mathrm{Al}_{2} \mathrm{O}_{3}$ are also widebandgap insulators, we cannot exclude that impeded incorporation of oxygen at the surface (mechanism ii) may have a similar or additional effect. However, in this case one may also expect a similar effect from YSZ, as the high initial resistance of $\mathrm{SrTiO}_{3} / \mathrm{YSZ}$ heterostructures (Supplementary Fig. 3) indicates that only an extremely small concentration of conduction band electrons is present in the YSZ layer. We conclude, therefore, that mechanism iii is the most apparent explanation for the improved retention.

Regarding the voltage-time dilemma described in the introduction, one might question whether the incorporation of such layers for retention stabilization through slow oxygen migration might not lead to very slow switching times simultaneously. The extreme nonlinearity of oxygen migration with respect to the applied electric field and temperature, however, can yield sufficiently fast switching times even for large oxygen-migration activation energies due to Joule heating ${ }^{58}$.

We would like to emphasize that only with $\mathrm{Al}_{2} \mathrm{O}_{3}$, the retention-stabilization layer with the highest oxygen-migration enthalpy used here, we were able to introduce stable retention with $10 \mathrm{~mA}$ current compliance. We concur that this current is still extremely high regarding the application in integrated circuits. However, the current compliances necessary to achieve resistive switching in micrometre-sized single-crystalline $\mathrm{SrTiO}_{3}$ devices are always on this order of magnitude $\mathrm{e}^{11,16,46}$ and might be attributed to the inherently low defect density. To achieve stable retention in the $\mathrm{SrTiO}_{3}$-based devices shown in Figs 1 and 2, a much higher current compliance of $50 \mathrm{~mA}$ was applied, but the majority of devices still showed retention failure after short times. We conclude, therefore, that the intentional introduction of suitable retention-stabilization layers reduces the current necessary for the desired retention performance markedly. 
This power reduction has very beneficial effects: major structural changes such as phase separations or severe lattice distortions are commonly observed for $\mathrm{SrTiO}_{3}$ thin-film devices (compare Fig. 2 and refs 9,46). Within the switching filament shown in Fig. 5a, the structure in both the $\mathrm{SrTiO}_{3}$ and the $\mathrm{Al}_{2} \mathrm{O}_{3}$ shows no evident difference compared with the surrounding of the filament. The absence of structural changes of the active layers during switching is desirable for future memory and logic devices, yielding more uniform and reproducible devices. This is apparent from the much more uniform retention behaviour of $\mathrm{SrTiO}_{3} / \mathrm{Al}_{2} \mathrm{O}_{3}$ heterostructures (Fig. 5b) compared with the $\mathrm{SrTiO}_{3}$ thin-film devices in Fig. 1 .

By purely empirical studies and without paying special attention to oxygen transport properties of the materials under consideration, several groups already observed improved device performance in memristive devices with inserted $\mathrm{Al}_{2} \mathrm{O}_{3}$ layers, such as improved uniformity of $\mathrm{HfO}_{x^{-}}$and $\mathrm{TaO}_{x}$-based devices ${ }^{59,60}$, improved memory windows in $\mathrm{TiO}_{x}$-based devices ${ }^{55}$ and improved retention in $\mathrm{GdO}_{x}$-based devices ${ }^{61}$. On the basis of these observations, it can be assumed that our concept of the rationally designed retention-stabilization layer can be extrapolated from our model system to technologically more relevant systems.

In conclusion, we utilized the model system for valencechange-resistive switching, $\mathrm{SrTiO}_{3}$, to elucidate the impact of rationally designed retention-stabilization layers for stable retention in memristive devices. We demonstrated that spatially confined valence changes between $\mathrm{Ti}^{3+}$ and $\mathrm{Ti}^{4+}$ within a switching filament are responsible for the resistance change upon the application of electrical stimuli, as well as for the retention failure. While the low oxygen-migration enthalpy of $\mathrm{SrTiO}_{3}$ causes the frequently observed retention failure of the LRS, Jouleheating-induced local phase separation within the switching filament can stabilize the retention due to the formation of a $\mathrm{SrTiO}_{3} / \mathrm{SrO}$ interface, which inhibits fast reoxidation due to retarded oxygen transport through the SrO layer. We used this insight to derive a design rule for failure-resistant memristive devices: incorporation of oxide materials with slow oxygen diffusion, as the retention-stabilization layer protects the switching filaments from reoxidation and leads to significantly prolongated retention times, as was demonstrated here with $\mathrm{SrO}$ and $\mathrm{Al}_{2} \mathrm{O}_{3}$ layers. Utilizing good oxygen conductors such as YSZ as the retention-stabilization layer, on the other hand, yields frequent retention failure. In addition to improved retention, more uniform device performance can be achieved with $\mathrm{Al}_{2} \mathrm{O}_{3}$ retention-stabilization layers due to reduced heat generation compared with devices without intentional retention-stabilization layers.

\footnotetext{
Methods

Sample fabrication. Two-nanometre single-crystalline undoped $\mathrm{SrTiO}_{3}$ thin films were fabricated via pulsed laser deposition on $0.5-\mathrm{wt} \% \mathrm{Nb}: \mathrm{SrTiO}_{3}$ substrates (CrysTec). The single-crystalline $\mathrm{SrTiO}_{3}$ target was ablated by a $\mathrm{KrF}$ excimer laser $(\lambda=248 \mathrm{~nm})$ with a repetition rate of $5 \mathrm{~Hz}$ and a spot size of $2 \mathrm{~mm}^{2}$ at a target-tosubstrate distance of $44 \mathrm{~mm}$. The laser fluence was $1.4 \mathrm{~J} \mathrm{~cm}^{-2}$, yielding nearly stoichiometric films ${ }^{49}$. All samples were grown in an oxygen atmosphere of $0.1 \mathrm{mbar}$ at a substrate temperature of $800^{\circ} \mathrm{C}$. The film growth was monitored using reflection high-energy electron diffraction (akSA400 system).

$\mathrm{SrTiO}_{3} / \mathrm{SrO}$ heterostructures were obtained by subsequent in situ pulsed laser deposition of $2 \mathrm{~nm}$ undoped, slightly Sr-deficient $\mathrm{SrTiO}_{3}$ thin films (laser fluence $1.75 \mathrm{~J} \mathrm{~cm}^{-2}$, oxygen atmosphere of $0.1 \mathrm{mbar}$ ) and $1 \mathrm{~nm} \mathrm{SrO}$ (laser fluence $1.3 \mathrm{~J} \mathrm{~cm}^{-2}$, oxygen atmosphere of $10^{-7} \mathrm{mbar}$ ) on 0.5 -wt\% $\mathrm{Nb}_{\mathrm{SrTiO}}$ substrates (CrysTec). A single-crystalline $\mathrm{SrTiO}_{3}$ and a polycrystalline $\mathrm{SrO}_{2}$ target were used, with a repetition rate of $1 \mathrm{~Hz}$ and a spot size of $2 \mathrm{~mm}^{2}$ at a target-to-substrate distance of $44 \mathrm{~mm}$ for both layers.

$\mathrm{SrTiO}_{3} / \mathrm{YSZ}$ and $\mathrm{SrTiO}_{3} / \mathrm{Al}_{2} \mathrm{O}_{3}$ heterostructures were obtained similarly. After $\mathrm{SrTiO}_{3}$ deposition as described above, the samples were cooled down to room temperature before ex situ pulsed laser deposition of $\mathrm{YSZ}$ or $\mathrm{Al}_{2} \mathrm{O}_{3}$ at room temperature. For both cases, the laser fluence was $2.1 \mathrm{~J} \mathrm{~cm}^{-2}$, with a repetition rate
}

of $5 \mathrm{~Hz}$ and a spot size of $1.5 \mathrm{~mm}^{2}$ at a target-to-substrate distance of $60 \mathrm{~mm}$ in an oxygen atmosphere of $10^{-4}$ mbar.

For the top electrodes, a 30-nm Au layer was sputter deposited and structured into $10 \times 10-\mu \mathrm{m}^{2}$ electrode pads by optical lithography and reactive ion beam etching.

Electrical characterization. For electrical characterization, the top electrodes were contacted with tungsten whisker probes. The $\mathrm{Nb}: \mathrm{SrTiO}_{3}$ substrate served as an electrically grounded bottom electrode and was contacted by wire bonding. $I-V$ sweeps were performed with a Keithley 2611A SourceMeter. The different sweeps were performed using the following voltage cycles: $0 \mathrm{~V}$ to positive voltages $($ maximum $+3.75 \mathrm{~V}$ ) for forming and SET, $0 \mathrm{~V}$ to negative voltages (maximum $-5 \mathrm{~V}$ ) for RESET and $+0.5 \mathrm{~V}$ to $-0.5 \mathrm{~V}$ for read-out. The device resistance was obtained from the slope of a linear fit of the read-out sweeps between -0.1 and $+0.1 \mathrm{~V}$. The step size was $20 \mathrm{mV}$ and the holding time before measurement was $5 \mathrm{~ms}$; the current compliance for the forming step and the SET process was between 1 and $50 \mathrm{~mA}$, as indicated in the main text. During the RESET sweeps no current compliance was necessary. After the forming process, some of the cells were kept in the LRS and other were switched back into HRS for subsequent impedance spectroscopy and spectromicroscopic analysis. Impedance spectroscopy was performed on virgin cells and representative cells in the HRS and LRS using an HP 4291B RF Impedance/Material Analyzer with a sampling voltage of $300 \mathrm{mV}$ and a frequency range from 20 to $10^{6} \mathrm{~Hz}$. The impedance data were fitted with a commercially available software Zplot/Zview (Scibner Associates Inc.). An equivalent circuit consisting of a parallel circuit of a capacitor and a resistor was used to fit the data over the entire frequency range.

Top electrode delamination. For spectromicroscopic analysis, the top electrodes were delaminated under ultra-high vacuum conditions with an adhesive copper tape after sputter deposition of a homogeneous $30 \mathrm{~nm}$ Au layer onto the entire sample. The delaminated sample was transferred into the XPEEM chamber under ultra-high vacuum conditions.

Spectromicroscopy. All XPEEM experiments have been performed at the NanoESCA beamline at Elettra synchrotron laboratory (Trieste, Italy) using the endstation described elsewhere ${ }^{62}$. The complete removal of the Au top electrode is checked by recording of the spatially resolved Au 4f XPS spectrum on the former device area, and for all presented devices the absence of any gold signal has been verified (with the exception of a small amount of Au residue for the device presented in Supplementary Fig. 1). The total energy resolution determined from both the spectrometer broadening (the pass energy $50 \mathrm{eV}$ and the entrance slit $1 \mathrm{~mm}$ ) and the photon bandwidth was $200 \mathrm{meV}$ for photoemission spectra. Various series of images were taken at increasing electron kinetic energies with a step size of $0.1 \mathrm{eV}$; the photon energy was $200 \mathrm{eV}$. Core level photoemission spectra were extracted from the resulting image stack in different regions of interest. Similarly, image stacks with increasing photon energy were recorded for absorption spectra, using a step size of $0.05 \mathrm{eV}$ for the Ti L-edge and $0.2 \mathrm{eV}$ for the O-K-edge. The electron energy above the Fermi edge $E-E_{\mathrm{F}}$ was $5.2 \mathrm{eV}$. The image stacks were analysed and spectra were extracted using the IGOR Pro software. The fitting of the core level photoelectron spectra was performed after principal component analysis with CasaXPS Version 2.3.16 (ref. 63). The components were constrained to have the same peak position and width for each fit. False colour maps for the Sr 3d core levels were created by fitting the spectra at each pixel using the peak model described in Fig. 2. For X-ray absorption, false colour maps were created by fitting the spectra at each pixel using model absorption spectra for $\mathrm{Ti}^{3+}$ and $\mathrm{Ti}^{4+}$, or $\mathrm{O}$ in $\mathrm{SrO}$ and $\mathrm{O}$ in $\mathrm{SrTiO}_{3}$, respectively. The colour scale represents the spectral contribution of the $\mathrm{Ti}^{3+}$ signal. For all image stacks, the linear, vertical energy dispersion of the beamline exit slit and the parabolic, vertical energy dispersion of the NanoESCA energy filter were corrected as described in ref. 64 .

TEM analysis. Cross-section TEM specimens were cut using focused ion beam milling with a Ga ion beam at $30 \mathrm{kV}$ beam energy and polished at $5 \mathrm{kV}$ on an FEI Helios NanoLab 400S, followed by thinning and cleaning in a Fischione 1040 Nanomill with an Ar ion beam at energy of $900 \mathrm{eV}$ for thinning and $500 \mathrm{eV}$ for cleaning, with filament current of $0.21 \mathrm{~mA}$ and specimen tilt angles of $\pm 10^{\circ}$. HAADF-STEM imaging was conducted at $200 \mathrm{kV}$ with an FEI Titan G2 80-200

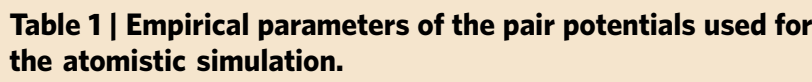

\begin{tabular}{lcccc} 
& $\boldsymbol{D}_{\mathbf{i j}} / \mathbf{e V}$ & $\boldsymbol{a}_{\mathbf{i j}} / \AA^{-\mathbf{1}}$ & \multicolumn{1}{c}{$\boldsymbol{r}_{\mathbf{o}} / \boldsymbol{\AA}$} & $\boldsymbol{C}_{\mathbf{i j}} / \mathbf{e V} \AA^{\mathbf{1 2}}$ \\
\hline $\mathrm{Sr}^{1.2+}-\mathrm{O}^{1.2-}$ & 0.019623 & 1.886000 & 3.328330 & 3.0 \\
$\mathrm{Ti}^{2.4+}-\mathrm{O}^{1.2-}$ & 0.024235 & 2.254703 & 2.708943 & 1.0 \\
$\mathrm{O}^{1.2-}-\mathrm{O}^{1.2-}$ & 0.042395 & 1.379316 & 3.618701 & 22.0 \\
\hline
\end{tabular}


ChemiSTEM microscope equipped with a high-brightness Schottky field emission electron gun, a probe $C_{\mathrm{S}}$ corrector and a Super-X EDX system. A nonlinear filtering algorithm was used for denoising of the HAADF-STEM images ${ }^{65}$. One-dimensional averaging of the HAADF-STEM images was carried out with a homemade package written in the Digital Micrograph scripting language.

Computational methods. Static atomistic simulations of the $\mathrm{SrTiO}_{3} / \mathrm{SrO}$ heterostructure performed in this study employed empirical pair potentials of the form

$$
V_{i j}=\frac{z_{i} z_{j} e^{2}}{r}+D_{i j}\left\{\left[1-e^{-a_{i j}\left(r-r_{0}\right)}\right]^{2}-1\right\}+\frac{C_{i j}}{r^{12}} .
$$

The first term is a coulomb term, which accounts for the long-range electrostatic interactions within the system. The second term is a Morse potential, which accounts for the short-range interactions, and the third term is a repulsive term, which corrects for Pauli repulsion and becomes important when performing high-temperature simulations. The empirical parameters of the potentials are obtained from the work of Pedone et al. ${ }^{66}$ and are listed in Table 1.

All degrees of freedom of the initial simulation cell of the $\mathrm{SrTiO}_{3} / \mathrm{SrO}$ heterostructure are optimized to a minimum energy configuration before the defect calculations. Defect calculations for determining vacancy and saddle-point configuration energies were performed employing the Mott-Littleton approach ${ }^{67}$. All simulations were implemented within the GULP code $^{68}$.

\section{References}

1. Waser, R. \& Aono, M. Nanoionics-based resistive switching memories. Nat. Mater. 6, 833-840 (2007).

2. Sawa, A. Resistive switching in transition metal oxides. Mater. Today 11, 28-36 (2008).

3. Waser, R., Dittmann, R., Staikov, G. \& Szot, K. Redox-based resistive switching memories - nanoionic mechanisms, prospects, and challenges. Adv. Mater. 21, 2632-2663 (2009).

4. Yang, J. J. et al. Memristive switching mechanism for metal/oxide/metal nanodevices. Nat. Nanotechnol. 3, 429-433 (2008).

5. Wong, H. S. P. et al. Metal-oxide RRAM. Proc. IEEE 100, 1951-1970 (2012).

6. Linn, E., Rosezin, R., Kügeler, C. \& Waser, R. Complementary resistive switches for passive nanocrossbar memories. Nat. Mater. 9, 403-406 (2010).

7. Janousch, M. et al. Role of oxygen vacancies in $\mathrm{Cr}$-doped $\mathrm{SrTiO} 3$ for resistancechange memory. Adv. Mater. 19, 2232-2235 (2007).

8. Lenser, C. et al. Probing the oxygen vacancy distribution in resistive switching Fe-SrTiO3 metal-insulator-metal-structures by micro-x ray absorption near-edge structure. J. Appl. Phys. 111, 076101 (2012).

9. Dittmann, R. et al. Scaling potential of local redox processes in memristive SrTiO3 thin-film devices. Proc. IEEE 100, 1979-1990 (2012).

10. Koehl, A. et al. Evidence for multifilamentary valence changes in resistive switching $\mathrm{SrTiO} 3$ devices detected by transmission X-ray microscopy. APL Mater. 1, 042102 (2013).

11. Lenser, C. et al. Insights into nanoscale electrochemical reduction in a memristive oxide: the role of three-phase boundaries. Adv. Funct. Mater. 24, 4466-4472 (2014).

12. Moreno, C. et al. Reversible resistive switching and multilevel recording in $\mathrm{La} 0.7 \mathrm{Sr} 0.3 \mathrm{MnO} 3$ thin films for low cost nonvolatile memories. Nano Lett. 10, 3828-3835 (2010).

13. Rana, V. \& Waser, R. in Memristors Memristive Systems (ed. Tetzlaff, R.) 223-251 (Springer, 2014).

14. Noman, M., Jiang, W., Salvador, P. A., Skowronski, M. \& Bain, J. A. Computational investigations into the operating window for memristive devices based on homogeneous ionic motion. Appl. Phys. A Mater. Sci. Process 102, 877-883 (2011).

15. Dong-Wook, K., Minji, G., Eunsongyi, L., Ahrum, S. \& El, M. B. Doping-level dependences of switching speeds and the retention characteristics of resistive switching $\mathrm{Pt} / \mathrm{SrTiO}_{3}$ junctions. J. Korean Phys. Soc. 57, 1432 (2010).

16. Raab, N., Bäumer, C. \& Dittmann, R. Impact of the cation-stoichiometry on the resistive switching and data retention of $\mathrm{SrTiO} 3$ thin films. AIP Adv. 5, 047150 (2015).

17. Yang, M. et al. Direct evidences of filamentary resistive switching in $\mathrm{Pt} / \mathrm{Nb}$-doped SrTiO3 junctions. J. Appl. Phys. 115, 134505 (2014).

18. Choi, D., Lee, D., Sim, H., Chang, M. \& Hwang, H. Reversible resistive switching of SrTiOx thin films for nonvolatile memory applications. Appl. Phys. Lett. 88, 082904 (2006).

19. Ninomiya, T. et al. Conductive filament scaling of TaOx bipolar ReRAM for improving data retention under low operation current. IEEE Trans. Electron Devices 60, 1384-1389 (2013).

20. Ninomiya, T. et al. Improvement of data retention during long-term use by suppressing conductive filament expansion in TaOx bipolar-ReRAM. IEEE Electron Device Lett. 34, 762-764 (2013).
21. Gao, B. et al. in IEEE Internatinal Electron Devices Meeting (Oxide-Based RRAM Switching Mechanism: A New Ion-Transport-Recombination Model) 1-4 (San Francisco, CA, USA, 2008).

22. Gao, B. et al. in Proceedings of the European Solid State Device Research Conference ESSDERC (Oxide-Based RRAM: Physical Based Retention Projection) 392-395 (Sevilla, Spain, 2010).

23. Lee, H. Y. et al. in IEEE International Electron Devices Meeting (Low Power and High Speed Bipolar Switching with a Thin Reactive Ti Buffer Layer in Robust HfO2 Based RRAM) 1-4 (San Francisco, CA, USA, 2008).

24. Chen, Y. S. et al. in IEEE International Electron Devices Meeting (Highly Scalable Hafnium Oxide Memory with Improvements of Resistive Distribution and Read Disturb Immunity) 1-4 (Baltimore, MD, USA, 2009).

25. Yu, S. M., Chen, Y. Y., Guan, X. M., Wong, H. S. P. \& Kittl, J. A. A Monte Carlo study of the low resistance state retention of $\mathrm{HfOx}$ based resistive switching memory. Appl. Phys. Lett. 100, 43507 (2012).

26. Chen, Y. Y. et al. Endurance/retention trade-off on $\mathrm{HfO} 2 \backslash$ metal cap $1 \mathrm{~T} 1 \mathrm{R}$ bipolar RRAM. IEEE Trans. Electron Devices 60, 1114-1121 (2013).

27. Wei, Z. et al. in IEEE International Electron Devices Meeting (Highly Reliable TaOx ReRAM and Direct Evidence of Redox Reaction Mechanism) 1-4 (San Francisco, CA, USA, 2008).

28. Goux, L. et al. Evidences of electrode-controlled retention properties in Ta2O5-based resistive-switching memory cells. ECS Solid State Lett. 3, Q79-Q81 (2014).

29. Ninomiya, T. et al. Conductive filament scaling of TaOx bipolar ReRAM for long retention with low current operation. Symp. VLSI Technol. 48, 73-74 (2012).

30. Waser, R. Bulk Conductivity and defect chemistry of acceptor-doped strontium titanate in the quenched state. J. Am. Ceram. Soc. 74, 1934-1940 (1991).

31. De Souza, R. A., Metlenko, V., Park, D. \& Weirich, T. E. Behavior of oxygen vacancies in single-crystal SrTiO3: equilibrium distribution and diffusion kinetics. Phys. Rev. B 85, 174109 (2012).

32. Schie, M., Waser, R. \& De Souza, R. a. A simulation study of oxygen-vacancy behavior in strontium titanate: beyond nearest-neighbor interactions. J. Phys. Chem. C 118, 15185-15192 (2014).

33. Moos, R. \& Hardtl, K. H. Defect chemistry of donor-doped and undoped strontium titanate ceramics between $1000^{\circ}$ and $1400^{\circ} \mathrm{C}$. J. Am. Ceram. Soc. 80, 2549-2562 (1997).

34. Denk, I., Münch, W. \& Maier, J. Partial conductivities in $\mathrm{SrTiO}_{3}$ : bulk polarization experiments, oxygen concentration cell measurements, and defectchemical modeling. J. Am. Ceram. Soc. 78, 3265-3272 (1995).

35. Gömann, K. et al. Sr diffusion in undoped and La-doped SrTiO3 single crystals under oxidizing conditions. Phys. Chem. Chem. Phys. 7, 2053-2060 (2005).

36. Metlenko, V. et al. Do dislocations act as atomic autobahns for oxygen in the perovskite oxide SrTiO3? Nanoscale 6, 12864-12876 (2014).

37. Muenstermann, R., Menke, T., Dittmann, R. \& Waser, R. Coexistence of filamentary and homogeneous resistive switching in Fe-doped SrTiO3 thin-film memristive devices. Adv. Mater. 22, 4819-4822 (2010).

38. Koehl, A. et al. Evidence for multifilamentary valence changes in resistive switching SrTiO3 devices detected by transmission X-ray microscopy. APL Mater. 1, 042102 (2013).

39. Abbate, M. et al. Soft-x-ray-absorption studies of the location of extra charges induced by substitution in controlled-valence materials. Phys. Rev. B 44, 5419-5422 (1991).

40. Strachan, J. P. et al. Direct identification of the conducting channels in a functioning memristive device. Adv. Mater. 22, 3573-3577 (2010).

41. Miao, F. et al. Anatomy of a nanoscale conduction channel reveals the mechanism of a high-performance memristor. Adv. Mater. 23, 5633-5640 (2011).

42. McLeod, J. A. et al. Band gaps and electronic structure of alkaline-earth and post-transition-metal oxides. Phys. Rev. B 81, 245123 (2010).

43. Meyer, R., Szot, K. \& Waser, R. Restructuring the surface region of donor doped SrTiO 3 single crystals under oxidizing conditions. Ferroelectrics 224, 323-329 (1999).

44. Meyer, R., Waser, R., Helmbold, J. \& Borchardt, G. Cationic surface segregation in donor-doped $\mathrm{SrTiO} 3$ under oxidizing conditions. J. Electroceramics $\mathbf{9}$, 101-110 (2002).

45. Rahmati, B. et al. Oxidation of reduced polycrystalline $\mathrm{Nb}$-doped $\mathrm{SrTiO}$ : characterization of surface islands. Surf. Sci. 595, 115-126 (2005).

46. Lenser, C. et al. Formation and movement of cationic defects during forming and resistive switching in $\mathrm{SrTiO}_{3}$ thin film devices. Adv. Funct. Mater. doi:10.1002/adfm.201500851 (2015).

47. Szot, K. et al. Formation of micro-crystals on the (100) surface of $\mathrm{SrTiO} 3$ at elevated temperatures. Surf. Sci. 460, 112-128 (2000).

48. Tanuma, S., Powell, C. J. \& Penn, D. R. Proposed formula for electron inelastic mean free paths based on calculations for 31 materials. Surf. Sci. 192, L849-L857 (1987).

49. Baeumer, C. Surface termination conversion during SrTiO3 thin film growth revealed by X-ray photoelectron spectroscopy. Sci. Rep. 5, 11829 (2015). 
50. Meyer, R. et al. in 9th Annual Non-Volatile Memory Technology Symposium (Oxide Dual-Layer Memory Element for Scalable Non-Volatile Cross-Point Memory Technology) 1-5 (Pacific Grove, CA, USA, 2008).

51. Merkle, R. \& Maier, J. Oxygen incorporation into Fe-doped SrTiO3: mechanistic interpretation of the surface reaction. Phys. Chem. Chem. Phys. 4, 4140-4148 (2002).

52. De Souza, R. A. A universal empirical expression for the isotope surface exchange coefficients $\left(\mathrm{k}^{*}\right)$ of acceptor-doped perovskite and fluorite oxides. Phys. Chem. Chem. Phys. 8, 890 (2006).

53. De Souza, R. A. et al. Oxygen diffusion in nanocrystalline yttria-stabilized zirconia: the effect of grain boundaries. Phys. Chem. Chem. Phys. 10, 2067-2072 (2008).

54. Heuer, A. H. Oxygen and aluminum diffusion in $\alpha$-Al2O3: How much do we really understand? J. Eur. Ceram. Soc. 28, 1495-1507 (2008)

55. Zhang, H., Aslam, N., Reiners, M., Waser, R. \& Hoffmann-Eifert, S. Atomic layer deposition of $\mathrm{TiOx} / \mathrm{Al} 2 \mathrm{O} 3$ bilayer structures for resistive switching memory applications. Chem. Vap. Deposition 20, 1-9 (2014).

56. Chen, Y. Z. et al. Room temperature formation of high-mobility twodimensional electron gases at crystalline complex oxide interfaces. Adv. Mater. 26, 1462-1467 (2014).

57. Chen, Y. Z. et al. A high-mobility two-dimensional electron gas at the spinel/perovskite interface of $\gamma$-Al2O3/SrTiO3. Nat. Commun. 4, 1371 (2013).

58. Menzel, S. et al. Origin of the ultra-nonlinear switching kinetics in oxide-based resistive switches. Adv. Funct. Mater. 21, 4487-4492 (2011).

59. Yu, S., Wu, Y., Chai, Y., Provine, J. \& Wong, H. S. P. in Int. Symp. VLSI Technol. Syst. Appl. Proc. 106-107 (Hsinchu, Taiwan, 2011).

60. Kim, Y.-B. et al. in Symposium on VLSI Technology (Bi-layered RRAM with Unlimited Endurance and Extremely Uniform Switching) 52-53 (Honolulu, HI, USA, 2011).

61. Wang, J. C., Jian, D. Y., Ye, Y. R. \& Chang, L. C. Platinum-aluminum alloy electrode for retention improvement of gadolinium oxide resistive switching memory. Appl. Phys. A 113, 37-40 (2013).

62. Wiemann, C. et al. A new nanospectroscopy tool with synchrotron radiation: NanoESCA@Elettra. e-J. Surf. Sci. Nanotechnol. 9, 395-399 (2011).

63. Kim, H. et al. Doping efficiency of single and randomly stacked bilayer graphene by iodine adsorption. Appl. Phys. Lett. 105, 011605 (2014).

64. Zagonel, L. F. et al. Orientation-dependent work function of in situ annealed strontium titanate. J. Phys. Condens. Matter 21, 314013 (2009).

65. Du, H. A nonlinear filtering algorithm for denoising HR(S)TEM micrographs. Ultramicroscopy 151, 62-67 (2015).

66. Pedone, A., Malavasi, G., Menziani, M. C., Cormack, A. N. \& Segre, U. A new self-consistent empirical interatomic potential model for oxides, silicates, and silica-based glasses. J. Phys. Chem. B 110, 11780-11795 (2006).
67. Mott, N. F. \& Littleton, M. J. Conduction in polar crystals. I. Electrolytic conduction in solid salts. Trans. Faraday Soc. 34, 485-499 (1938).

68. Gale, J. D. GULP: a computer program for the symmetry-adapted simulation of solids. J. Chem. Soc. Faraday Trans. 93, 629-637 (1997).

\section{Acknowledgements}

Funding from the DFG (German Science Foundation) within the collaborative research center SFB 917 'Nanoswitches' is gratefully acknowledged. C.B. and R.D. also acknowledge funding from the W2/W3 program of the Helmholtz association. K.S. acknowledges funding from the Initiative and Networking Fund of the German Helmholtz Association, Helmholtz Virtual Institute VH-VI-442 MEMRIOX. We thank Maximilian Kruth for preparing the TEM lamellae. C.B. would like to thank Dr. Paul Meuffels and Dr. Stephan Menzel for insightful discussions.

\section{Author contributions}

C.B. and R.D. conceived and designed the experiments. C.B., C.S., H.D., K.S., V.F., B.A. and P.M. fabricated the samples and performed the experiments. A.R. and R.A.D.S. constructed the model system for oxygen migration and performed the static simulations. H.D. carried out the HAADF-STEM imaging. H.D., C.-L.J. and J.M. analysed the HAADF-STEM images. C.B. wrote the manuscript. C.M.S., R.W and R.D. supervised the research. All authors discussed the results and commented on the manuscript.

\section{Additional information}

Supplementary Information accompanies this paper at http://www.nature.com/ naturecommunications

Competing financial interests: The authors declare no competing financial interests.

Reprints and permission information is available online at http://npg.nature.com/ reprintsandpermissions/

How to cite this article: Baeumer, C. et al. Spectromicroscopic insights for rational design of redox-based memristive devices. Nat. Commun. 6:8610 doi: $10.1038 /$ ncomms9610 (2015).

This work is licensed under a Creative Commons Attribution 4.0 International License. The images or other third party material in this article are included in the article's Creative Commons license, unless indicated otherwise in the credit line; if the material is not included under the Creative Commons license, users will need to obtain permission from the license holder to reproduce the material To view a copy of this license, visit http://creativecommons.org/licenses/by/4.0/ 